\title{
$N$-Soliton Solutions of the Nonisospectral Generalized Sawada-Kotera Equation
}

\author{
Jian Zhou, Xiang-Gui Li, and Deng-Shan Wang \\ School of Applied Science, Beijing Information Science and Technology University, Beijing 100192, China \\ Correspondence should be addressed to Jian Zhou; keyan_zhou@126.com
}

Received 6 May 2014; Revised 10 August 2014; Accepted 20 August 2014; Published 1 September 2014

Academic Editor: Alkesh Punjabi

Copyright (c) 2014 Jian Zhou et al. This is an open access article distributed under the Creative Commons Attribution License, which permits unrestricted use, distribution, and reproduction in any medium, provided the original work is properly cited.

\begin{abstract}
The soliton interaction is investigated based on solving the nonisospectral generalized Sawada-Kotera (GSK) equation. By using Hirota method, the analytic one-, two-, three-, and $N$-soliton solutions of this model are obtained. According to those solutions, the relevant properties and features of line-soliton and bright-soliton are illustrated. The results of this paper will be useful to the study of soliton resonance in the inhomogeneous media.
\end{abstract}

\section{Introduction}

The Hirota method, originating from the work of Hirota in 1971 [1], is a powerful method for constructing solutions for integrable systems. The soliton theory is presented in several monographs and review papers (see $[2,3])$. In the literature, various approaches have been proposed to find a soliton solution for a given equation, for instance, the inverse scatting transform [4] and the Darboux transformation [5]. It is remarked that the Hirota method is very efficient for construction of soliton solutions.

The nonisospectral equations describe solitary waves in inhomogeneous media. Recently, much attention has been paid on the analytic solutions of the nonisospectral equations. Deng et al. [6] and Sun et al. $[7,8]$ develop a systematic procedure to find soliton solutions of the nonisospectral equations. Based on exact solutions, numerical methods can be presented well for the nonisospectral nonlinear problem [9-11].

Jiang considers the nonisospectral problem [12] by using the compatibility condition of Lax pairs. In our work, the bilinear form and $N$-soliton solutions will be considered for a generalized nonisospectral equation.

The nonisospectral generalized Sawada-Kotera (GSK) equation [12] is written as follows:

$$
\begin{aligned}
u_{t} & -u_{x x x x x}-5 u_{x} u_{x x}-5 u u_{x x x}-u^{2} u_{x} \\
& -5 u_{x x y}+5 \partial_{x}^{-1} u_{y y}-5 u u_{y}-5 u_{x} \partial_{x}^{-1} u_{y} \\
& -\omega\left(2 u+x u_{x}+3 y u_{y}\right)-a u_{x}-b u_{y}=0,
\end{aligned}
$$

where $\omega, a$, and $b$ are real constants. The Lax pair of (1) is

$$
\begin{gathered}
L=\partial_{x}^{3}+u \partial_{x}+\partial_{y}, \\
M=\partial_{t}+9 \partial_{x}^{5}+(15 u+3 \omega y+b) \partial_{x}^{3}+15 u_{x} \partial_{x}^{2} \\
+\left(10 u_{x x}+5 u^{2}-5 \partial_{x}^{-1} u_{y}+(3 \omega y+b) u\right. \\
-\omega x-a) \partial_{x} .
\end{gathered}
$$

The aim of this paper is to propose a simple method for construction $N$-soliton solutions. The main tool is the Hirota method. Then we apply the idea to the nonisospectral GSK equation.

This paper is organized as following: In Section 2, with the aid of symbolic computation, the bilinear form of (1) is obtained by use of Hirota method. Some special solutions are explicitly presented based on their bilinear form (4) and the soliton resonance is illustrated. The final section contains some discussion.

\section{Bilinear Form and $N$-Soliton Solutions}

Through the dependent variable transformation

$$
u=3(15-\sqrt{185})(\ln f)_{x x} .
$$


Equation (1) can be written in the bilinear form. Consider

$$
\begin{aligned}
& D_{x} D_{t} f \cdot f-D_{x}^{6} f \cdot f+D_{x}^{4} f \cdot f-5 D_{x}^{3} D_{y} f \cdot f \\
& \quad-(a+\omega x) D_{x}^{2} f \cdot f+(1-b-3 \omega y) D_{x} D_{y} f \cdot f \\
& \quad+5 D_{y}^{2} f \cdot f-2 \omega f f_{x}=0,
\end{aligned}
$$

where the $D$-operators [13] is defined by

$$
\begin{aligned}
& D_{x}^{m} D_{t}^{n} a(x, t) \cdot b(x, t) \\
& \quad=\left.\left(\frac{\partial}{\partial x}-\frac{\partial}{\partial x^{\prime}}\right)^{m}\left(\frac{\partial}{\partial t}-\frac{\partial}{\partial t^{\prime}}\right)^{n} a(x, t) b\left(x^{\prime}, t^{\prime}\right)\right|_{x=x^{\prime}, t=t^{\prime}} .
\end{aligned}
$$

The perturbation method consists of expanding $f$ with respect to a small parameter $\varepsilon$ to obtain

$$
f=1+\varepsilon f^{(1)}+\varepsilon^{2} f^{(2)}+\varepsilon^{3} f^{(3)}+\cdots,
$$

and then finding each coefficient $f^{(n)}$ successively for $n=$ $1,2,3, \ldots$

Substituting the expansion formula of $f$ into the bilinear equation (4) and arranging it at each order of $\varepsilon$, we have

$$
\begin{aligned}
\varepsilon: & f_{t x}^{(1)}-f_{x x x x x x}^{(1)}+f_{x x x x}^{(1)}-5 f_{x x x y}^{(1)}-(a+\omega x) f_{x x}^{(1)} \\
& +(1-b-3 \omega y) f_{x y}^{(1)}+5 f_{y y}^{(1)}-\omega f_{x}^{(1)}=0 \\
\varepsilon^{2}: & 2\left[f_{t x}^{(2)}-f_{x x x x x x}^{(2)}+f_{x x x x}^{(2)}-5 f_{x x x y}^{(2)}-(a+\omega x) f_{x x}^{(2)}\right. \\
& \left.+(1-b-3 \omega y) f_{x y}^{(2)}+5 f_{y y}^{(2)}-\omega f_{x}^{(2)}\right] \\
& +D_{x} D_{t} f^{(1)} \cdot f^{(1)}-D_{x}^{6} f^{(1)} \cdot f^{(1)}+D_{x}^{4} f^{(1)} \cdot f^{(1)} \\
& -5 D_{x}^{3} D_{y} f^{(1)} \cdot f^{(1)}-(a+\omega x) D_{x}^{2} f^{(1)} \cdot f^{(1)} \\
& +(1-b-3 \omega y) D_{x} D_{y} f^{(1)} \cdot f^{(1)} \\
& +5 D_{y}^{2} f^{(1)} \cdot f^{(1)}-2 \omega f^{(1)} \cdot f_{x}^{(1)}=0 \\
\varepsilon^{3}: & f_{t x}^{(3)}-f_{x x x x x x}^{(3)}+f_{x x x x}^{(3)}-5 f_{x x x y}^{(3)}-(a+\omega x) f_{x x}^{(3)} \\
& +(1-b-3 \omega y) f_{x y}^{(3)}+5 f_{y y}^{(3)}-\omega f_{x}^{(3)} \\
& +D_{x} D_{t} f^{(1)} \cdot f^{(2)}-D_{x}^{6} f^{(1)} \cdot f^{(2)}+D_{x}^{4} f^{(1)} \cdot f^{(2)} \\
& -5 D_{x}^{3} D_{y} f^{(1)} \cdot f^{(2)}-(a+\omega x) D_{x}^{2} f^{(1)} \cdot f^{(2)} \\
& +(1-b-3 \omega y) D_{x} D_{y} f^{(1)} \cdot f^{(2)}+5 D_{y}^{2} f^{(1)} \cdot f^{(2)} \\
& -\omega\left(f^{(1)} \cdot f_{x}^{(2)}+f_{x}^{(1)} \cdot f^{(2)}\right)=0 \\
& +(1)
\end{aligned}
$$

let us choose

$$
f^{(1)}=e^{\xi_{1}}
$$

where $\xi_{1}=-k_{1}(t) x+k_{1}^{3}(t) y-(a / \omega) k_{1}(t)+(b / 3 \omega) k_{1}^{3}(t)+$ $(9 / 5 \omega) k_{1}^{5}(t)+\xi_{1}^{0}$.

Since substituting this into the left-hand side of (7) gives

$$
k_{1, t}(t)=\omega k_{1}(t)
$$

then (11) is an ordinary differential system and it can be solved exactly. The solutions of (11) are written as

$$
k_{1}(t)=c_{1} e^{\omega t}
$$

Therefore, we are able to choose $f^{(j)}=0, j=2,3, \ldots$. This shows that the expansion of $f$ may be truncated as the finite sum

$$
f=1+f^{(1)}=1+e^{\xi_{1}}
$$

Substituting (13) into (3), the one-soliton solution of the nonisospectral GSK equation (1) can be obtained

$$
u=\frac{3(15-\sqrt{185}) k_{1}^{2}(t)}{4} \operatorname{sech}^{2}\left(\frac{\xi_{1}}{2}\right)
$$

Here $u$ is the one-soliton solution. By the form of the solution (14), one can see that the one-soliton travels with a timedependent top trace

$$
\xi_{1}=0
$$

In fact, the top trace of the solution (14) is a line with timedependent slope. Equation (14) provides a line-soliton with the following time-dependent amplitude:

$$
A=\frac{3(15-\sqrt{185}) k_{1}^{2}(t)}{4} .
$$

Figures 1 and 2 describe the different amplitudes of the one-soliton solution at $t=1$ and $t=2$.

We begin here by finding a two-soliton solution. It is a solution describing the interaction of two solitons.

To this end, we choose the solution to the linear differential equation (7) to be

$$
f^{(1)}=e^{\xi_{1}}+e^{\xi_{2}}
$$

where $\xi_{j}=-k_{j}(t) x+k_{j}^{3}(t) y-(a / \omega) k_{j}(t)+(b / 3 \omega) k_{j}^{3}(t)+$ $(9 / 5 \omega) k_{j}^{5}(t)+\xi_{j}^{0}$ for $j=1,2$.

Substituting (17) into the left-hand side of (7), we have

$$
k_{j, t}(t)=\omega k_{j}(t), \quad(j=1,2) .
$$

The solutions of (18) are written as

$$
k_{j}(t)=c_{j} e^{\omega t}, \quad(j=1,2) .
$$

We here set that

$$
f^{(2)}=e^{\xi_{1}+\xi_{2}+\theta_{12}}
$$




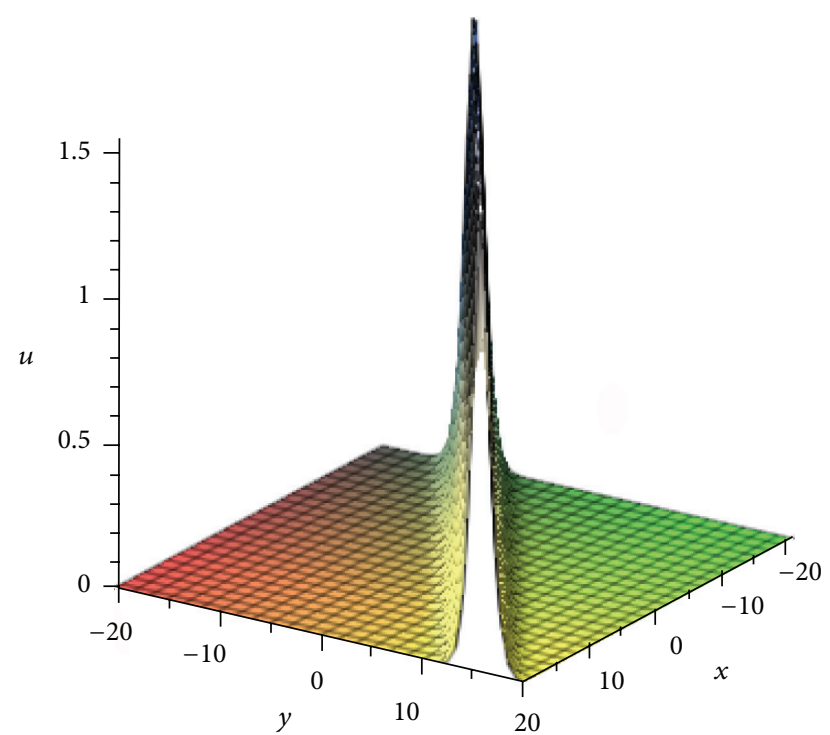

FIGURE 1: The shape and motion of the one-soliton solution for $\omega=$ $-0.8, a=1, b=1, c_{1}=1, \xi_{1}^{0}=1, t=1$.

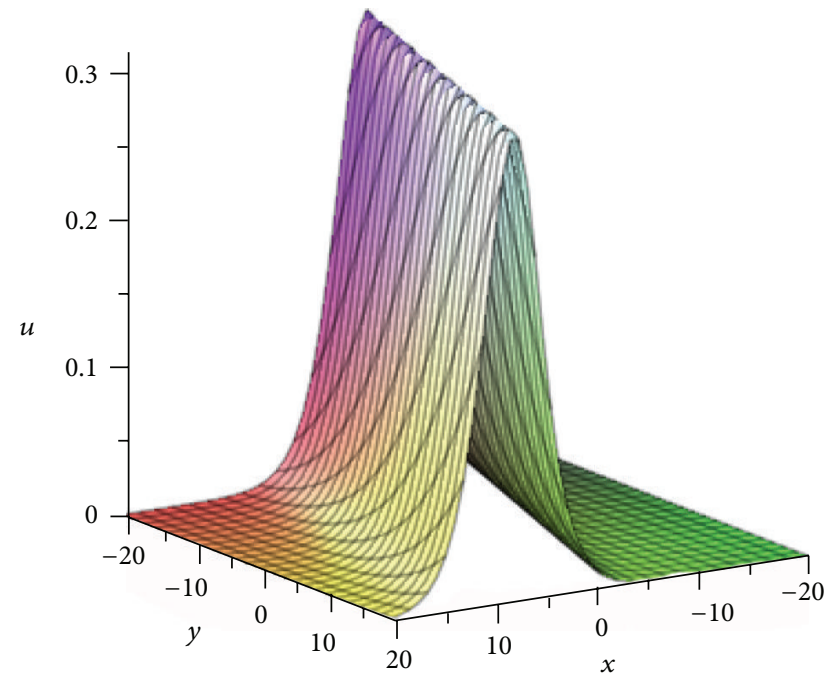

FIGURE 2: The shape and motion of the one-soliton solution for $\omega=$ $-0.8, a=1, b=1, c_{1}=1, \xi_{1}^{0}=1, t=2$.

From (20), we might assume that the relations $e^{\theta_{12}}=F(t)$. Equation (20) may also be written as

$$
f^{(2)}=F(t) e^{\xi_{1}+\xi_{2}}
$$

Substituting (17), (21) into the left-hand side of (8) and using (18), we have

$$
\begin{gathered}
\frac{\partial F(t)}{\partial t}-3 k_{1}(t) k_{2}(t)\left[k_{1}(t)+k_{2}(t)\right] F(t) \\
+\frac{3 k_{1}(t) k_{2}(t)\left[k_{1}(t)-k_{2}(t)\right]^{2}}{\left[k_{1}(t)+k_{2}(t)\right]}=0 .
\end{gathered}
$$

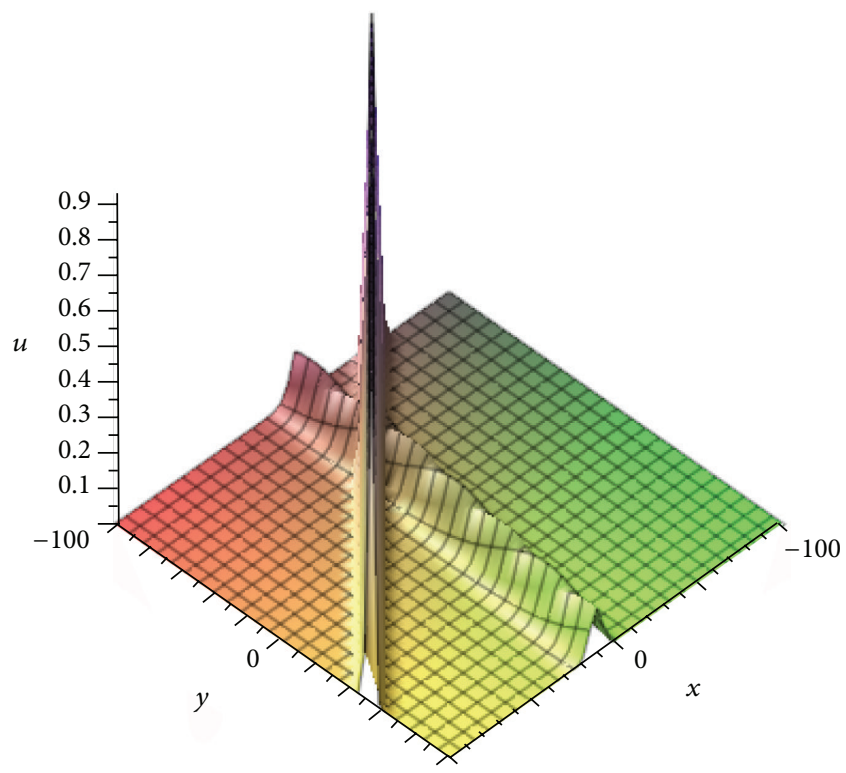

FIGURE 3: The shape and motion of the two-soliton solutions for $\omega=$ $-1, a=1, b=1, c_{1}=1, c_{2}=2, \xi_{1}^{0}=1, \xi_{2}^{0}=1, t=2$.

Substitution of (19) into (22) gives

$$
F(t)=\frac{\left[k_{1}(t)-k_{2}(t)\right]^{2}}{\left[k_{1}(t)+k_{2}(t)\right]^{2}}=e^{\theta_{12}} .
$$

The coefficient $e^{\theta_{12}}$ obtained in (23), which are similar to the SK equation (see [14]), can also be KdV type.

Therefore, we are able to choose $f^{(j)}=0, j=3,4, \ldots$. The two-soliton solutions are obtained by (3) in which $f$ is defined as

$$
f=1+f^{(1)}+f^{(2)}=1+e^{\xi_{1}}+e^{\xi_{2}}+e^{\xi_{2}+\xi_{2}+\theta_{12}} .
$$

Its shape and motion are shown in Figures 3 and 4.

In Figures 3 and 4, the line-soliton characters are shown in two-soliton solutions, where the black areas denote zero value and the white lines denote bright-soliton. In this case, the amplitudes and slopes of the two-soliton will vary with time and this time-dependent property comes from the effects of inhomogeneous media.

Let us choose

$$
f^{(1)}=e^{\xi_{1}}+e^{\xi_{2}}+e^{\xi_{3}}
$$

where $\xi_{j}=-k_{j}(t) x+k_{j}^{3}(t) y-(a / \omega) k_{j}(t)+(b / 3 \omega) k_{j}^{3}(t)+$ $(9 / 5 \omega) k_{j}^{5}(t)+\xi_{j}^{0}$ for $j=1,2,3$.

Substituting (25) into the left-hand side of (7), we have

$$
k_{j, t}(t)=\omega k_{j}(t), \quad(j=1,2,3) .
$$

The solutions of (26) are written as

$$
k_{j}(t)=c_{j} e^{\omega t}, \quad(j=1,2,3) .
$$




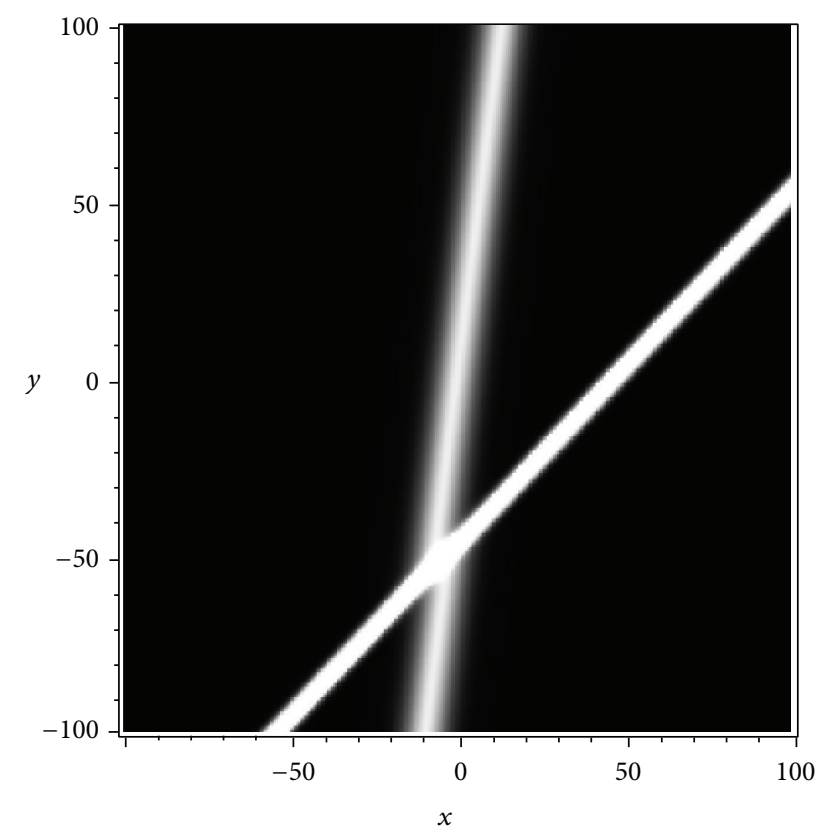

FIgURE 4: The density plots of two-soliton resonance for the nonisospectral GSK equation with parameters $\omega=-1, a=1, b=1$, $c_{1}=1, c_{2}=2, \xi_{1}^{0}=1, \xi_{2}^{0}=1, t=2$.

\section{We here set that}

$$
f^{(2)}=e^{\xi_{1}+\xi_{2}+\theta_{12}}+e^{\xi_{1}+\xi_{3}+\theta_{13}}+e^{\xi_{2}+\xi_{3}+\theta_{23}}
$$

Substitution of (25), (28) into (8) gives

$$
\begin{aligned}
e^{\theta_{12}} & =\frac{\left[k_{1}(t)-k_{2}(t)\right]^{2}}{\left[k_{1}(t)+k_{2}(t)\right]^{2}}, \\
e^{\theta_{13}} & =\frac{\left[k_{1}(t)-k_{3}(t)\right]^{2}}{\left[k_{1}(t)+k_{3}(t)\right]^{2}}, \\
e^{\theta_{23}} & =\frac{\left[k_{2}(t)-k_{3}(t)\right]^{2}}{\left[k_{2}(t)+k_{3}(t)\right]^{2}} .
\end{aligned}
$$

Let

$$
f^{(3)}=e^{\xi_{1}+\xi_{2}+\xi_{3}+\theta_{123}} .
$$

Substituting (25), (28), and (30) into (9), one obtains

$$
e^{\theta_{123}}=\frac{\left[k_{1}(t)-k_{2}(t)\right]^{2}\left[k_{1}(t)-k_{3}(t)\right]^{2}\left[k_{2}(t)-k_{3}(t)\right]^{2}}{\left[k_{1}(t)+k_{2}(t)\right]^{2}\left[k_{1}(t)+k_{3}(t)\right]^{2}\left[k_{2}(t)+k_{3}(t)\right]^{2}} .
$$

Therefore, we are able to choose $f^{(j)}=0, j=4,5, \ldots$. The three-soliton solutions are obtained by (3) in which $f$ is defined as

$$
\begin{aligned}
f= & 1+f^{(1)}+f^{(2)}+f^{(3)} \\
= & 1+e^{\xi_{1}}+e^{\xi_{2}}+e^{\xi_{3}}+e^{\xi_{1}+\xi_{2}+\theta_{12}}+e^{\xi_{1}+\xi_{3}+\theta_{13}} \\
& +e^{\xi_{2}+\xi_{3}+\theta_{23}}+e^{\xi_{1}+\xi_{2}+\xi_{3}+\theta_{123}}
\end{aligned}
$$

The nonisospectral GSK equation [12] has been shown to be integrable. It can be represented as the compatibility condition in the $\operatorname{Lax}$ form $[L, M]=0$. Therefore, it would be reasonable to continue to find the $N$-soliton solutions $(N>$ 3 ) with the help of symbolic computation (see [15]).

This process can be extended to the four-soliton solutions, and so on. Generally, the $N$-soliton solutions are expressed as

$$
f=\sum \exp \left[\sum_{j=1}^{N} \mu_{j} \xi_{j}+\sum_{j<l}^{(N)} \theta_{j l} \mu_{j} \mu_{l}\right],
$$

where the coefficients $\theta_{j l}$ and $\xi_{j}$ are defined by

$$
\begin{gathered}
\theta_{j l}=\frac{\left[k_{j}(t)-k_{l}(t)\right]^{2}}{\left[k_{j}(t)+k_{l}(t)\right]^{2}}, \\
\xi_{j}=-k_{j}(t) x+k_{j}^{3}(t) y-\frac{a}{\omega} k_{j}(t)+\frac{b}{3 \omega} k_{j}^{3}(t) \\
+\frac{9}{5 \omega} k_{j}^{5}(t)+\xi_{j}^{0}, \quad j=1,2, \ldots, N,
\end{gathered}
$$

respectively.

In formula (33), the first $\sum$ means a summation over all possible combinations of $\mu_{1}=0,1, \mu_{2}=0,1, \ldots, \mu_{N}=0,1$, and $\sum_{j<l}^{(N)}$ means a summation over all possible pairs $(j, l)$ chosen from the set $\{1,2, \ldots, N\}$, with the condition that $j<l$.

Substituting (33) into (3), we obtain the N-soliton solutions for the nonisospectral GSK equation.

\section{Conclusion}

In this paper, we have obtained the $N$-soliton solutions of the nonisospectral GSK equation by the Hirota method. Under transformation (3), (1) has been transformed into bilinear form (4) directly. Based on formula (33), N-soliton solutions have been constructed. A KdV-type solution has also been obtained. Soliton resonance and interaction for (1) can be regarded as the combination of the effects of various variable coefficients, as shown in Figures 1-3. Effects of the line-soliton, bright-soliton, and soliton resonance have been summarized. Finally, according to Figure 4, the possible applications of soliton resonance in the inhomogeneous media have been discussed.

\section{Conflict of Interests}

The authors declare that there is no conflict of interests regarding the publication of this paper.

\section{Acknowledgments}

This work is supported by National Natural Science Foundation of China under Grant nos. 11171032, 11271362, and 11375030 and Beijing special project from Beijing education committee. The third author is supported by Beijing Natural Science Foundation under Grant no. 1132016 and Beijing Nova program no. Z131109000413029. 


\section{References}

[1] R. Hirota, "Exact solution of the korteweg-de vries equation for multiple collisions of solitons," Physical Review Letters, vol. 27, no. 18, pp. 1192-1194, 1971.

[2] L. Pitaevskii and S. Stringari, Bose-Einstein Condensation, Oxford University, New York, NY, USA, 2003.

[3] D.-S. Wang and X.-G. Li, "Localized nonlinear matter waves in a Bose-Einstein condensate with spatially inhomogeneous two- and three-body interactions," Journal of Physics B: Atomic, Molecular and Optical Physics, vol. 45, no. 10, Article ID 105301, 2012.

[4] M. J. Ablowitz and H. Segur, Solitons and the Inverse Scattering Transform, SIAM, Philadelphia, Pa, USA, 1981.

[5] V. B. Matveev and M. A. Salle, Darboux Transformations and Solitons, Springer, Berlin, Germany, 1991.

[6] S. Deng, D. Zhang, and D. Chen, "Exact solutions for the nonisospectral Kadomtshev-Petviashvili equation," Journal of the Physical Society of Japan, vol. 74, no. 9, pp. 2383-2385, 2005.

[7] Y. Sun, J. Bi, and D. Chen, " $N$-soliton solutions and double Wronskian solution of the non-isospectral AKNS equation," Chaos, Solitons \& Fractals, vol. 26, no. 3, pp. 905-912, 2005.

[8] Y. Sun, "The non-isospectral modified Kadomtsev-Petviashvili equation with self-consistent sources and its coupled system," Applied Mathematics and Computation, vol. 217, no. 4, pp. 1639$1645,2010$.

[9] X. Li, W. Yan, and C. K. Chan, "Numerical schemes for Hamilton-Jacobi equations on unstructured meshes," Numerische Mathematik, vol. 94, no. 2, pp. 315-331, 2003.

[10] D. Hua and X. Li, "The finite element method for computing the ground states of the dipolar Bose-Einstein condensates," Applied Mathematics and Computation, vol. 234, pp. 214-222, 2014.

[11] S. Li, X. Li, and D. Hua, "A time-splitting and sine spectral method for dynamics of dipolar Bose-Einstein condensate," Advances in Mathematical Physics, vol. 2013, Article ID 517395, 7 pages, 2013.

[12] Z. Jiang, "Nonisospectral problems related to DS and other $(2+1)$-dimensional nonlinear evolution equations," Inverse Problems, vol. 9, no. 3, pp. L1-L8, 1993.

[13] R. Hirota, The Direct Methods in Soliton Theory, Cambridge University Press, Cambridge, UK, 2004.

[14] X. Lü, T. Geng, C. Zhang, H.-W. Zhu, X.-H. Meng, and B. Tian, "Multi-soliton solutions and their interactions for the $(2+1)$ dimensional sawada-kotera model with truncated painlevé expansion, hirota bilinear method and symbolic computation," International Journal of Modern Physics B, vol. 23, no. 25, pp. 5003-5015, 2009.

[15] R. Hirota, "Exact envelope-soliton solutions of a nonlinear wave equation," Journal of Mathematical Physics, vol. 14, no. 7, pp. 805-809, 1973. 


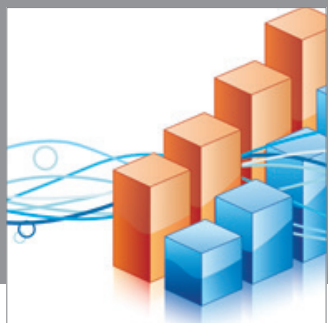

Advances in

Operations Research

mansans

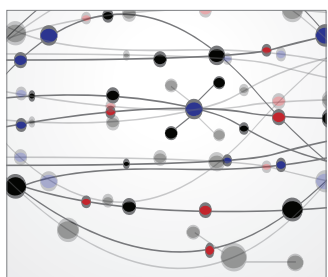

The Scientific World Journal
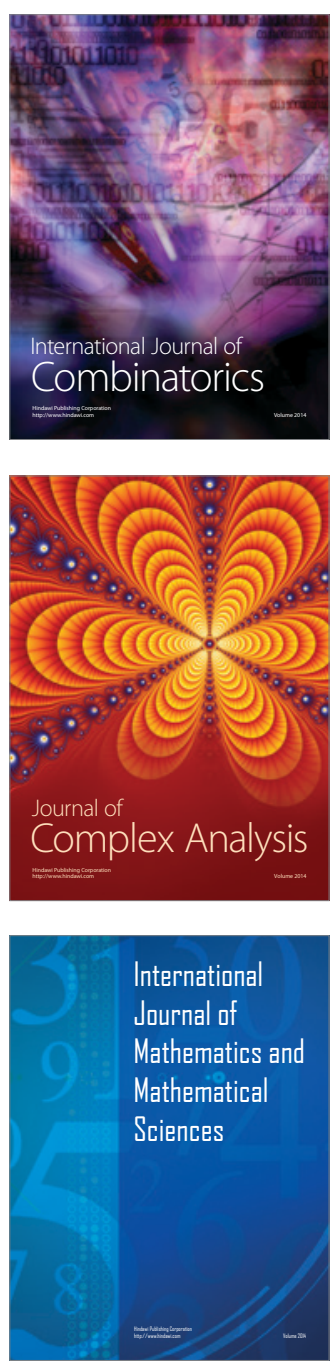
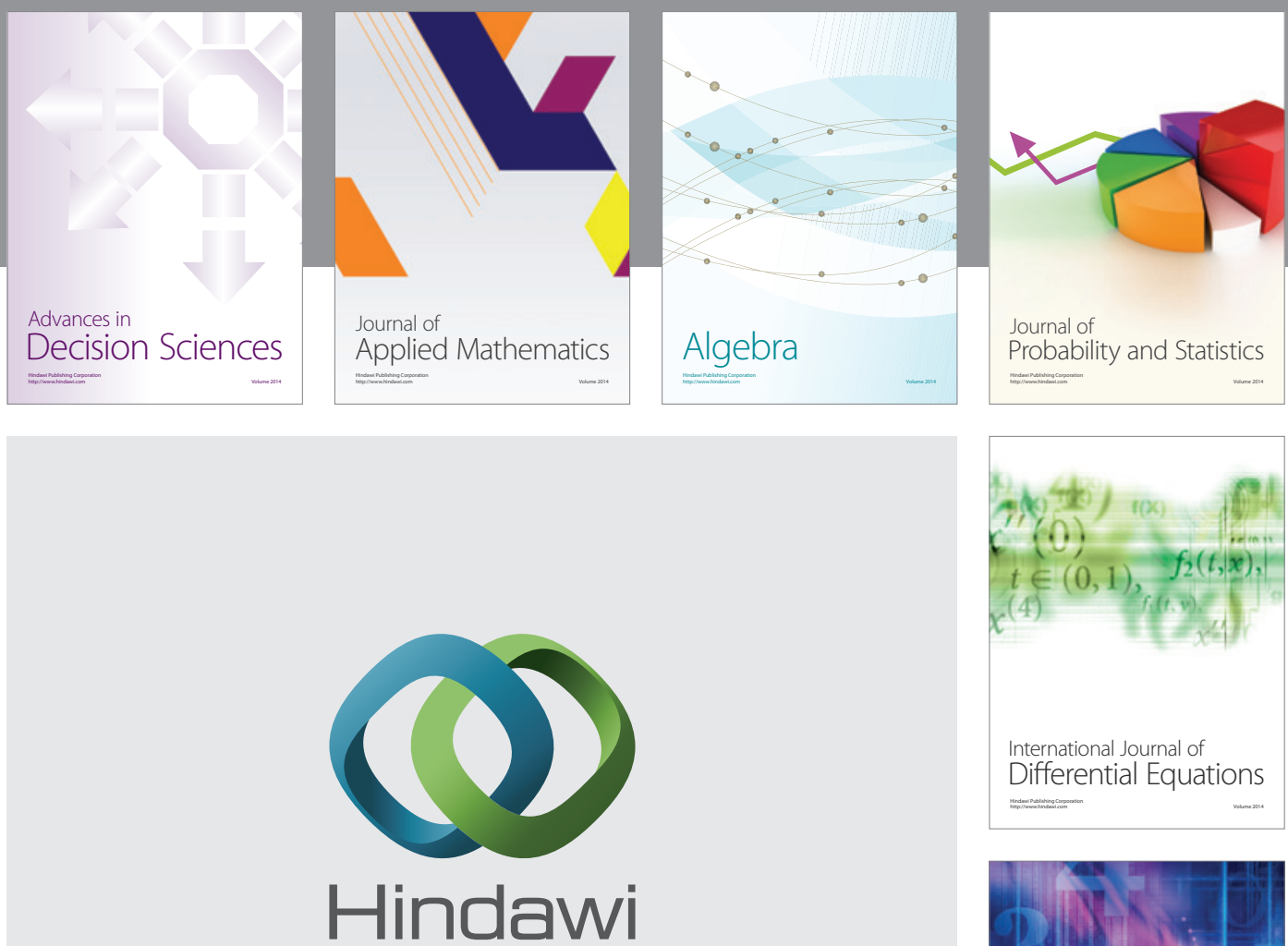

Submit your manuscripts at http://www.hindawi.com
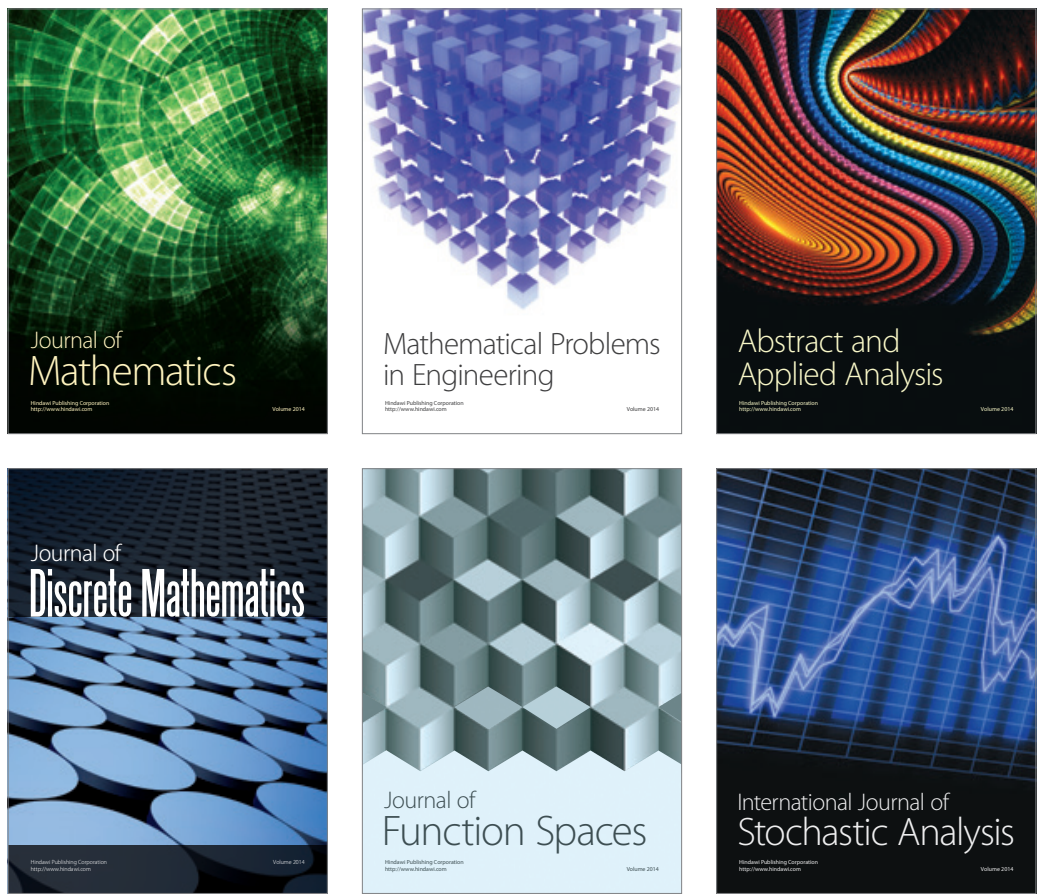

Journal of

Function Spaces

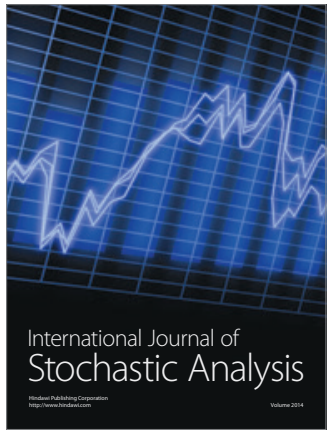

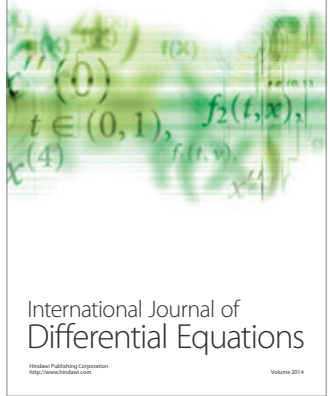
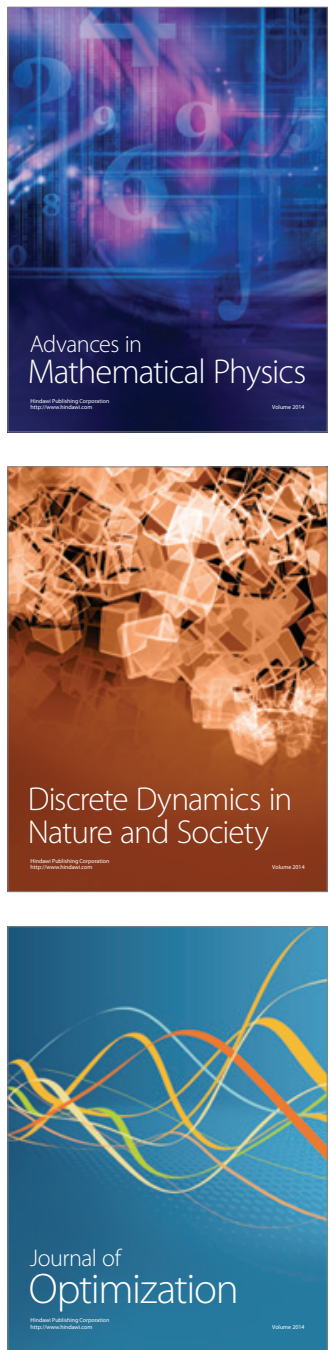